TITLE:

\title{
Three-dimensional diagnostics of dynamic behaviors of excited atoms in microplasma for plasma display panels
}

\author{
AUTHOR(S): \\ Tachibana, K; Mizokami, K; Kosugi, N; Sakai, T
}

\section{CITATION:}

Tachibana, K ... [et al]. Three-dimensional diagnostics of dynamic behaviors of excited atoms in microplasma for plasma display panels. IEEE TRANSACTIONS ON PLASMA SCIENCE 2003, 31(1): 68-73

\section{ISSUE DATE:}

2003-02

URL:

http://hdl.handle.net/2433/50086

\section{RIGHT:}

(c)2003 IEEE. Personal use of this material is permitted. However, permission to reprint/republish this material for advertising or promotional purposes or for creating new collective works for resale or redistribution to servers or lists, or to reuse any copyrighted component of this work in other works must be obtained from the IEEE. 


\title{
Three-Dimensional Diagnostics of Dynamic Behaviors of Excited Atoms in Microplasma for Plasma Display Panels
}

\author{
Kunihide Tachibana, Member, IEEE, Kaname Mizokami, Naoki Kosugi, and Tetsuo Sakai
}

Invited Paper

\begin{abstract}
A three-dimensional (3-D) observation cell with realistic ac-type structure and size was fabricated and measurements were done, for the first time, on the spatiotemporal behaviors of excited Xe atoms by using optical emission and laser absorption spectroscopic techniques. As a typical characteristic feature, it is seen that the discharge starts from the temporal anode edge, stretching toward the cathode, and a striated pattern of excited atoms appears on the anode side while a single broader peak distributes on the cathode side, traveling to its far end. In addition, the dependence on the applied voltage was clearly seen in the distribution of excited atoms, which shows the influence of the charge accumulation on the walls. With the two-dimensional projection images obtained from side and front views in both the emission and absorption measurements, an iterative method was tried preliminarily to derive the peak values of excited $\mathrm{Xe}$ atoms in the 3-D space.
\end{abstract}

Index Terms-Laser absorption spectroscopy, optical emission spectroscopy, plasma display panel (PDP), Xe excited atoms.

\section{INTRODUCTION}

A PLASMA display panel (PDP) is getting popular as a large wall-hanging flat display of diagonal size from 40 to $60 \mathrm{in}$. However, the improvement of luminous efficiency from a current typical value of $1-2$ to $5 \mathrm{~lm} / \mathrm{W}$ is a crucial problem for a PDP to be really available for home use. Various cell structures and operating conditions have been proposed and tested for the purpose, but the approaches up to now are mostly performed in trial-and-error manners. Therefore, it is difficult to get firm guiding principles toward the goal. Efforts to understand the physics of the microdischarges in a PDP cell have been done extensively by computer simulations [1]-[5], but those validity

Manuscript received August 8, 2002; revised November 5, 2002.

K. Tachibana is with the Department of Electronic Science and Engineering, Kyoto University, Kyoto 606-8501 Japan (e-mail: tatibana@kuee.kyoto-u.ac.jp).

K. Mizokami was with the Department of Electronic Science and Engineering, Kyoto University, Kyoto 606-8501 Japan. He is now with Matsushita Electric Industrial Co., Ltd., Osaka 569-1194, Japan (e-mail: PANB3561@ pas.mei.co.jp).

N. Kosugi is with the Plasma Display Division, Display Device Company, Matsushita Electric Industrial Co., Ltd., Osaka 569-1194 Japan (e-mail: kosugi@erl.mec.mei.co.jp).

T. Sakai is with the Display Research Laboratories Co. Ltd., Tokyo 158-0096

Japan (e-mail: drsakai@trio.plala.or.jp).

Digital Object Identifier 10.1109/TPS.2003.808882

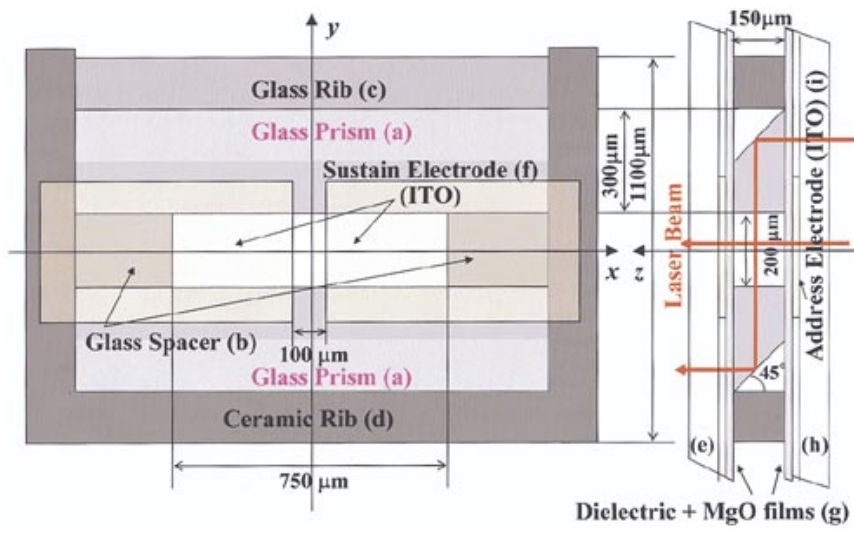

Fig. 1. Structure of ac-type PDP cell for front and side view observations.

should be checked by proper diagnostics on the characteristic behaviors of excited species [6], [7], the electric field [8], and the electron energy and density [9] in the small volume of a PDP discharge cell. We have developed a microscopic laser-absorption method [6], [7], which has a spatiotemporal resolution better than $20 \mu \mathrm{m}$ and $5 \mathrm{~ns}$, and applied it to the measurements of excited $\mathrm{Xe}\left(1 \mathrm{~s}_{4}, 1 \mathrm{~s}_{5}\right.$; hereafter Paschen notation is used) atoms leading to the vacuum ultraviolet (VUV) emissions in a front viewed two-dimensional (2-D) scheme, say in the $x-y$ plane (see Fig. 1). In addition, in order to make possible a comparison with the 2-D simulations mostly done in the $x-z$ plane, we have constructed a special panel for the cross-sectional view and performed a preliminary measurement [10].

However, as the size of the cell is becoming smaller and the electrodes are taking complicated patterns, a realistic three-dimensional (3-D) observation technique of spatiotemporal dynamic behaviors of excited atoms in a unit cell is being required for deeper understandings of the effects of cell designs to the luminous efficiency. This kind of precise 3-D analysis is difficult by simply combining the experimental results from the front-view and side-view panels, and a technique for the simultaneous observations in the same cell is essential. Thus, we have designed and manufactured a special panel for this purpose [11]. By using this 3-D observation cell, we have succeeded not only in the measurement of visible and near-IR emission characteristics by a charged-coupled device (CCD) camera, but also in the 
measurement of Xe atoms in the metastable $\left(1 \mathrm{~s}_{5}\right)$ and resonance $\left(1 \mathrm{~s}_{4}\right)$ states by laser absorption spectroscopy. Those characteristic 3-D behaviors of excited Xe atoms are presented here and the dependence on the applied voltage is going to be argued.

\section{EXPERIMENTAL}

\section{A. Test Panel Structure}

The structure of our present ac-type panel for the 3-D measurement is shown schematically in Fig. 1, with components labeled (a)-(i). It has a design similar to a realistic ac-type cell in the structure and size. The essential point to realize the 3-D observation was the idea of using transparent glass prisms (a) as barrier ribs. These were assembled with other rectangular glass spacers (b) and a backup glass rib (c) to fit into a ceramic outer rib (d), which was print-formed on the front glass plate (e). A pair of Indium-Tin-Oxide (ITO) sustain electrodes (f) were preformed on the front plate, which were over-coated successively by insulator film and $\mathrm{MgO}$ film (g). On the back glass plate (h), an addressing (or data) electrode (i) made of ITO was formed and covered by an insulator film. A mixture of $\mathrm{Xe}(5 \%)-\mathrm{Ne}$ was filled at a pressure of 500 torr. The minimum firing and sustain voltages of this cell were 300 and $180 \mathrm{~V}$, respectively. The sustain voltage pulses were applied to one of the electrodes with a width $\tau_{1}$ of $10 \mu$ s and period $T$ of $20.8 \mu \mathrm{s}$, and the same pulses were applied to the other electrode with a delay $\left(\tau_{1}+\tau_{2}\right)$ of $10.4 \mu$ s (see Fig. 8).

\section{B. Measuring Techniques}

For the emission measurement, a gated CCD camera equipped with an image intensifier and an objective lens of $20 \times$ magnification factor was used. The gate width applied to the CCD camera was set at $10 \mathrm{~ns}$ and the sampling time was scanned by a delay generator. For the simultaneous observation of front and side views, the focusing length was set in between the direct path from the discharge volume and the bent path through a prism as shown in Fig. 1. In the case of near infrared emission from $\mathrm{Xe}(2 \mathrm{p})$ levels, an optical bandpass filter with 20-nm bandwidth [full-width at half-maximum (FWHM)] centered at $820 \mathrm{~nm}$ was mounted in front of the CCD camera to get the spectral resolution.

The densities of Xe atoms in the $1 s_{5}$ and $1 s_{4}$ states were measured by the laser absorption technique using a diode laser tuned at the wavelengths of $823 \mathrm{~nm}\left(2 \mathrm{p}_{6}-1 \mathrm{~s}_{5}\right)$ and $828 \mathrm{~nm}\left(2 \mathrm{p}_{5}-1 \mathrm{~s}_{4}\right)$, respectively [6], [7]. In these measurements, the test panel was mounted on an optical microscope, and the spatial resolution better than $20 \mu \mathrm{m}$ was attained. The absolute density was derived from the integrated area of each spectral line by taking into account of the effects due to the hyperfine structures and the collisional line broadening and shift as was done previously [6], [7].

\section{RESULTS}

\section{A. Near IR Emission From Xe(2p) Atoms}

Spatiotemporal behaviors of near IR emission from excited $\mathrm{Xe}(2 \mathrm{p})$ atoms are shown in Figs. 2 and 3, which were taken as typical examples operated at low and high values of the applied

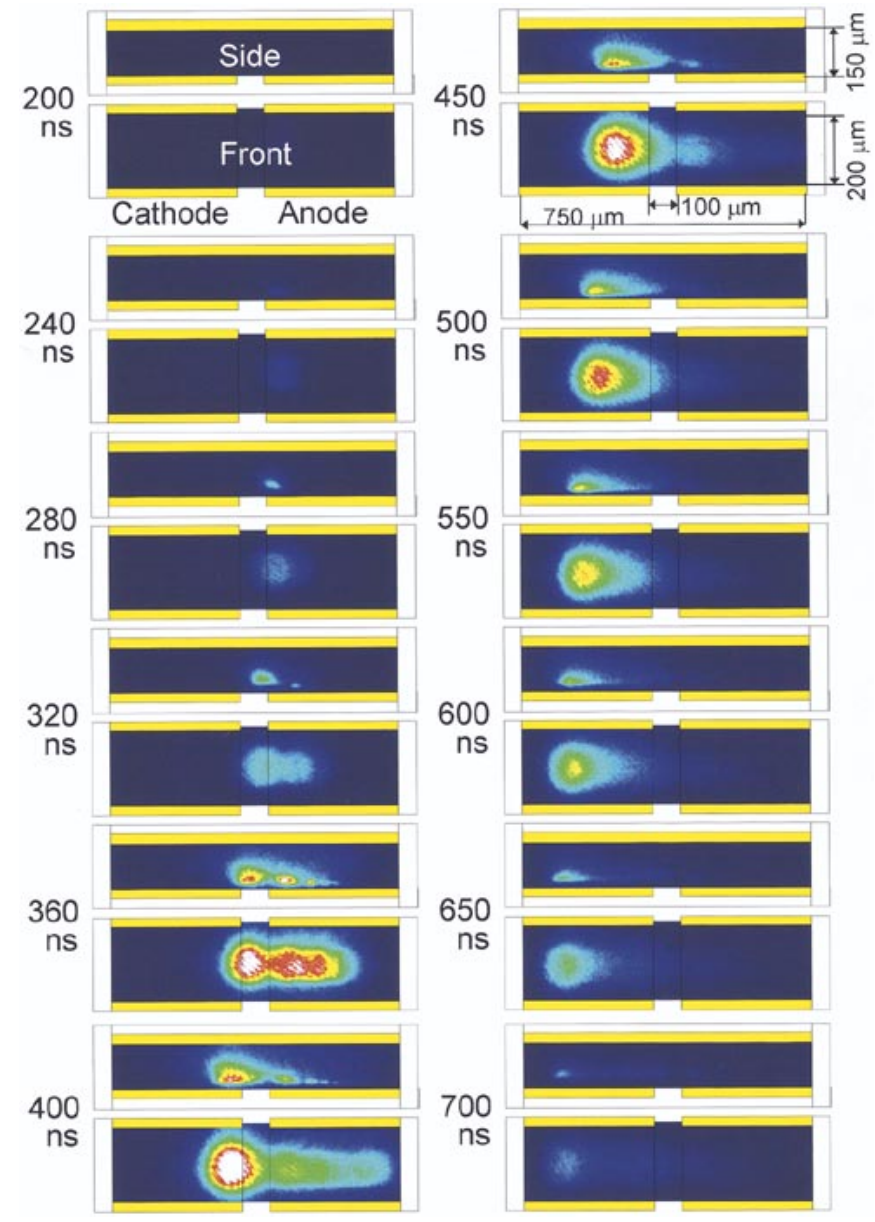

Fig. 2. Spatiotemporal behavior of near IR emission from $\mathrm{Xe}(2 \mathrm{p})$ atoms at low-voltage $(200 \mathrm{~V})$ mode measured by a CCD camera.

pulse voltage, i.e., 200 and $250 \mathrm{~V}$, respectively. The address electrode was kept at the ground level. At the low-voltage mode operation $(200 \mathrm{~V})$, the behavior was as expected from our previous measurements [7], [10]. The discharge started at near the temporal anode edge and extended toward the cathode. Then, a broader peak appeared on the cathode side while several narrower peaks appeared on the anode side, although these peaks were not fully seen in the side view image due to their location close to the electrode surface. The delay time of the discharge after the pulse voltage application was about $200 \mathrm{~ns}$, and the current waveform had a peak of $300 \mu \mathrm{A}$ and an FWHM of $150 \mathrm{~ns}$.

On the other hand, at the high-voltage case $(250 \mathrm{~V})$ it is clearly seen that the discharge was initiated between the temporal cathode and the address electrode after the falling-off of the preceding pulse and before the application of the succeeding pulse. This is a kind of self-erasing discharge, of which mechanism will be discussed later in detail. Just after the application of the succeeding pulse, the main discharge started and extended toward the temporal anode, but the spatial distribution of discharge volume bent in a $U$ shape toward the address electrode. It has been empirically known that in this so-called high-voltage mode the luminosity and the efficiency are improved substantially [12]. From the present observation, the reason may be attributed to the enhancement of the discharge volume with the influence of the charge accumulated on the address electrode as will be discussed in the following. 


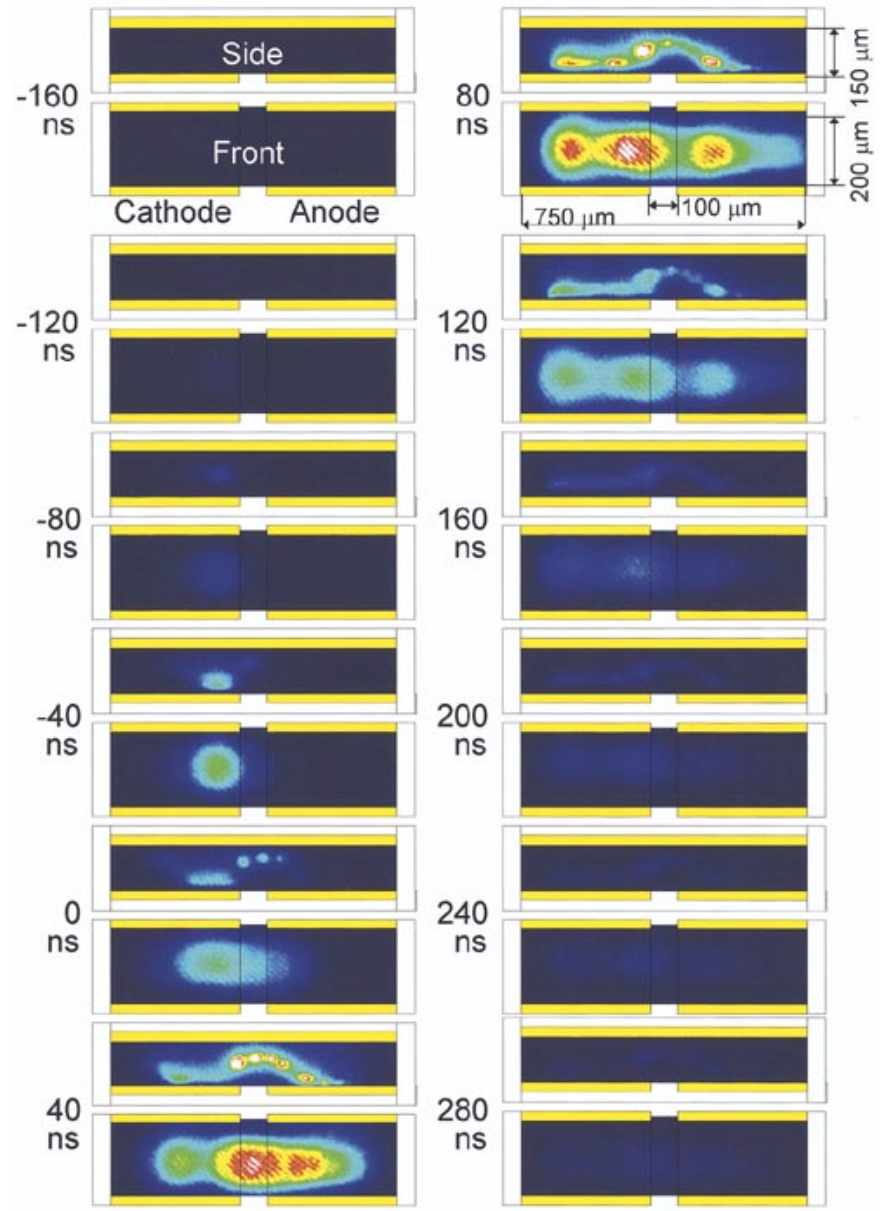

Fig. 3. Spatiotemporal behavior of near IR emission from $\mathrm{Xe}(2 \mathrm{p})$ atoms at high-voltage $(250 \mathrm{~V})$ mode measured by CCD camera.

The duration of the discharge, however, was shorter in this case since the larger current density caused the faster charging on the electrode surfaces to reduce the gap voltage.

\section{B. Behavior of $\mathrm{Xe}\left(1 s_{5}, 1 s_{4}\right)$ Atoms}

By using the laser absorption technique, we measured the spatiotemporal behavior of the excited Xe atoms in the metastable $\left(1 s_{5}\right)$ state. Figs. 4 and 5 show the results in a time sequence for the low-voltage and high-voltage operation modes, respectively, corresponding to the conditions for the emission measurement. The regions within about $25-\mu \mathrm{m}$ distance from the electrode and barrier rib surfaces could not be accessed by the probing laser beam. Therefore, those regions are not shown in the figures. Typical features in the spatial distribution in both the low-voltage and high-voltage modes are similar to the case of the emission results given previously. The major difference is in the longer decay of the $\mathrm{Xe}\left(1 \mathrm{~s}_{5}\right)$ atom density; it is governed by the loss rate mainly due to the three-body process leading to the $\mathrm{Xe}_{2} *$ excimer formation, which gives a lifetime of about 1 $\mu \mathrm{s}$ a lifetime of $\mathrm{Xe}\left(1 \mathrm{~s}_{5}\right)$ atoms of about $2 \mu \mathrm{s}$.

The temporal behavior of the total number of $\mathrm{Xe}\left(1 \mathrm{~s}_{5}\right)$ atoms in the anode-side and cathode-side volumes divided in the midplane are calculated from the experimental data and shown in Fig. 6 together with the summed value over the whole volume. It is seen from the figure that the number of excited atoms on

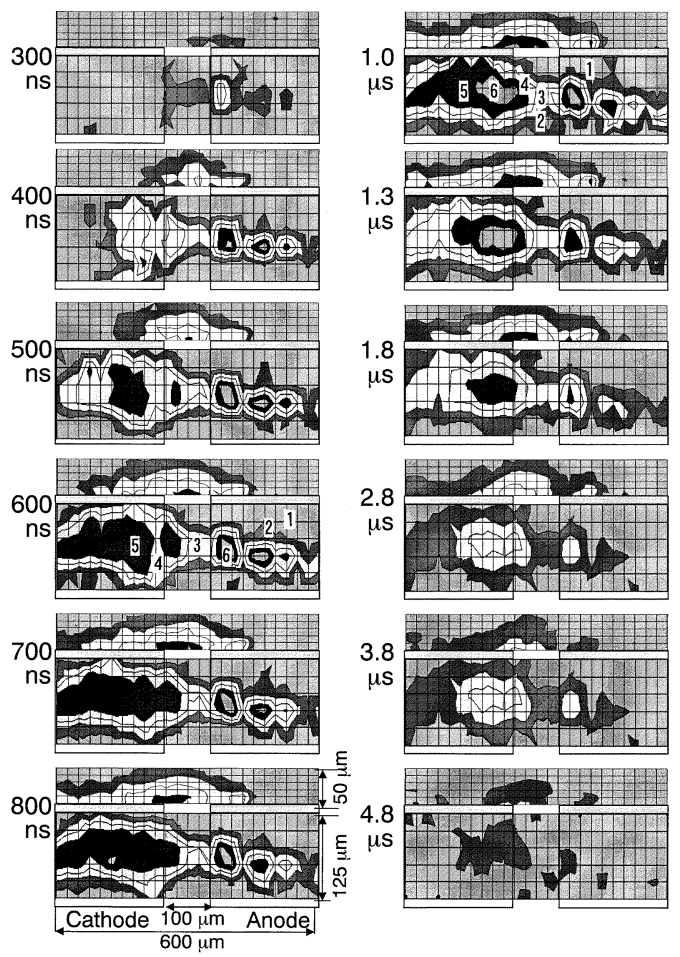

Fig. 4. Spatiotemporal behavior of $\mathrm{Xe}\left(1 \mathrm{~s}_{5}\right)$ atom density at low-voltage $(200 \mathrm{~V})$ mode measured by laser absorption spectroscopy (density scales are 1 : $0-0.4 ; 2: 0.4-0.8 ; 3: 0.8-1.2 ; 4: 1.2-1.6 ; 5: 1.6-2.0$; and $6: 2.0-2.4$ in units of $\left.10^{13} \mathrm{~cm}^{-3}\right)$.

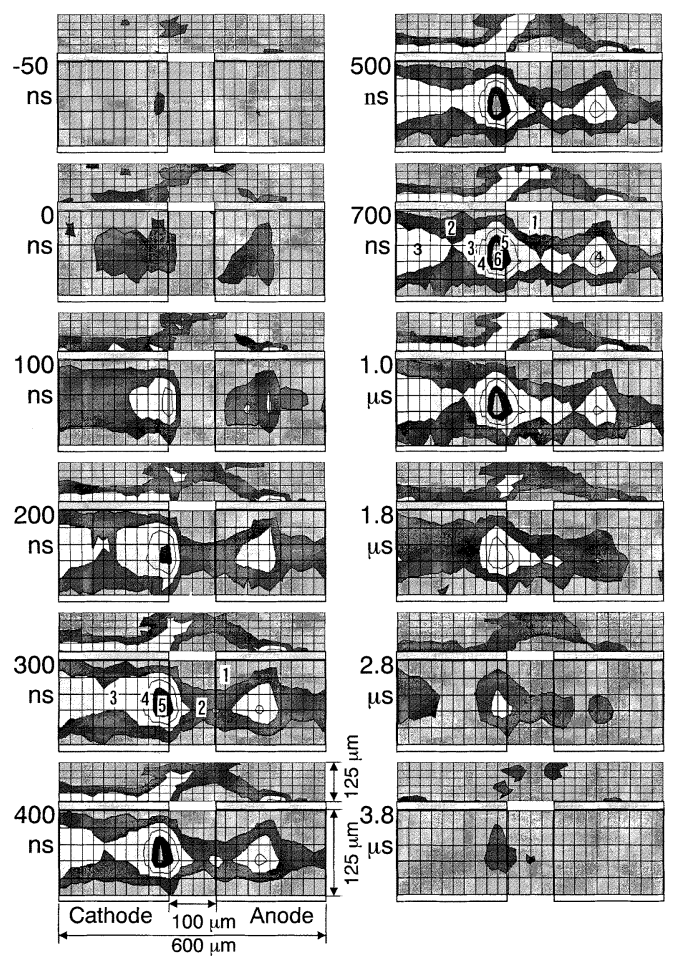

Fig. 5. Spatiotemporal behavior of $\mathrm{Xe}\left(1 \mathrm{~s}_{5}\right)$ atom density at high-voltage $(250 \mathrm{~V})$ mode measured by laser absorption spectroscopy (density scales are $1: 0-1.0 ; 2: 1.0-2.0 ; 3: 2.0-3.0 ; 4: 3.0-4.0 ; 5: 4.0-5.0$; and $6: 5.0-6.0$ in units of $10^{13} \mathrm{~cm}^{-3}$ ).

the cathode side is larger than that on the anode side. The total value at the high-voltage mode reaches almost twice the value of the low-voltage mode. Unfortunately, it was difficult to derive 

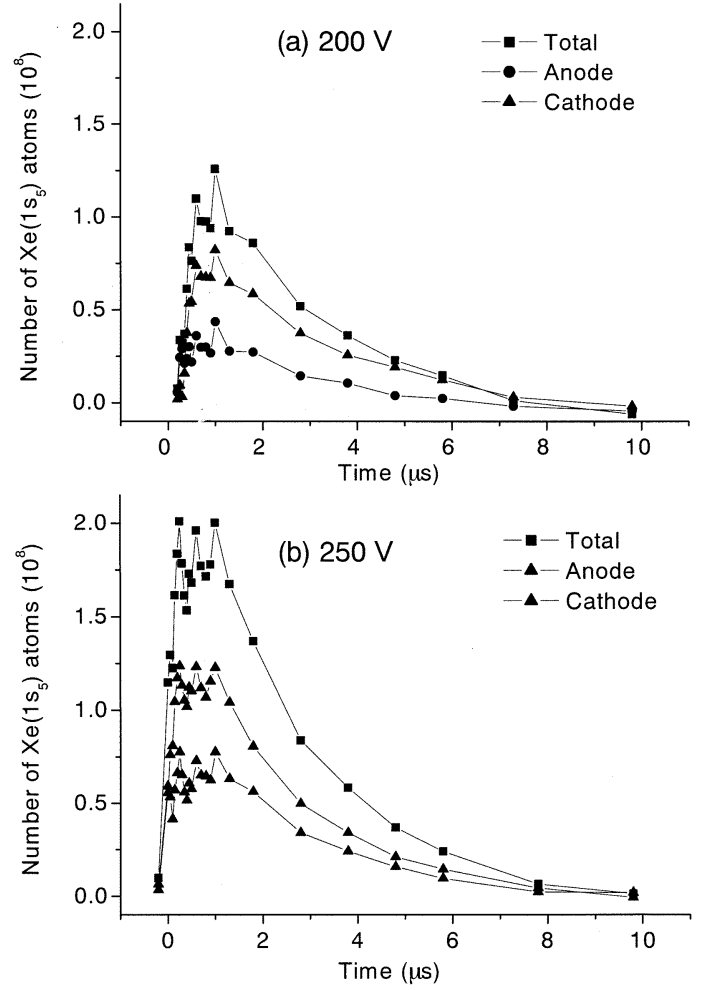

Fig. 6. Number of $\mathrm{Xe}\left(1 \mathrm{~s}_{5}\right)$ atoms in anode-side and cathode-side half-volumes and the grand total plotted as a function of time at (a) low-voltage $(200 \mathrm{~V})$ and (b) high-voltage $(250 \mathrm{~V})$ modes.

an accurate current waveform in the high-voltage mode since the displacement current overlapped severely with the conductive current due to the very small ignition delay as mentioned previously. If we assume the near IR emission waveform is proportional to the current waveform, the ratio of the production efficiency of metastable atoms in these two modes is estimated to be about 1.5, which suggests an improvement of the efficacy by $50 \%$ in the high-voltage mode.

Similar measurement was done for the density of $\mathrm{Xe}\left(1 \mathrm{~s}_{4}\right)$ atoms in the resonance state. The result at the low-voltage mode is shown in Fig. 7. As far as the characteristics in the spatial distribution it is similar to the result for $\mathrm{Xe}\left(1 \mathrm{~s}_{5}\right)$ atoms, although the data includes a larger noise due to the smaller absorption signal. However, the temporal behavior is different due to the relatively shorter effective lifetime $\tau$ of the $147 \mathrm{~nm}$ transition. The value of $\tau$ is estimated from the decay of the total number of $\mathrm{Xe}\left(1 \mathrm{~s}_{4}\right)$ atoms from a similar plot to Fig. 6 as about $0.5 \mu \mathrm{s}$. The peak density reaches $1.6 \times 10^{13} \mathrm{~cm}^{-3}$ as compared to the corresponding value of $2.2 \times 10^{13} \mathrm{~cm}^{-3}$ for $\mathrm{Xe}\left(1 \mathrm{~s}_{5}\right)$ atoms at the peak of the front view.

\section{DISCUSSION}

We will first try to explain the characteristic differences between the two operating modes. As shown schematically in Fig. 8, the wall-charge accumulation does affect the wall voltages at the sustain electrodes (denoted as $V_{1}$ and $V_{2}$ ) and the address electrode $\left(V_{3}\right)$. If the charge density accumulated on the address electrode surface is large enough and the voltage difference between the preceding temporal anode and the address electrode $\left(V_{1}-V_{3}\right)$ causes the electric field strength larger

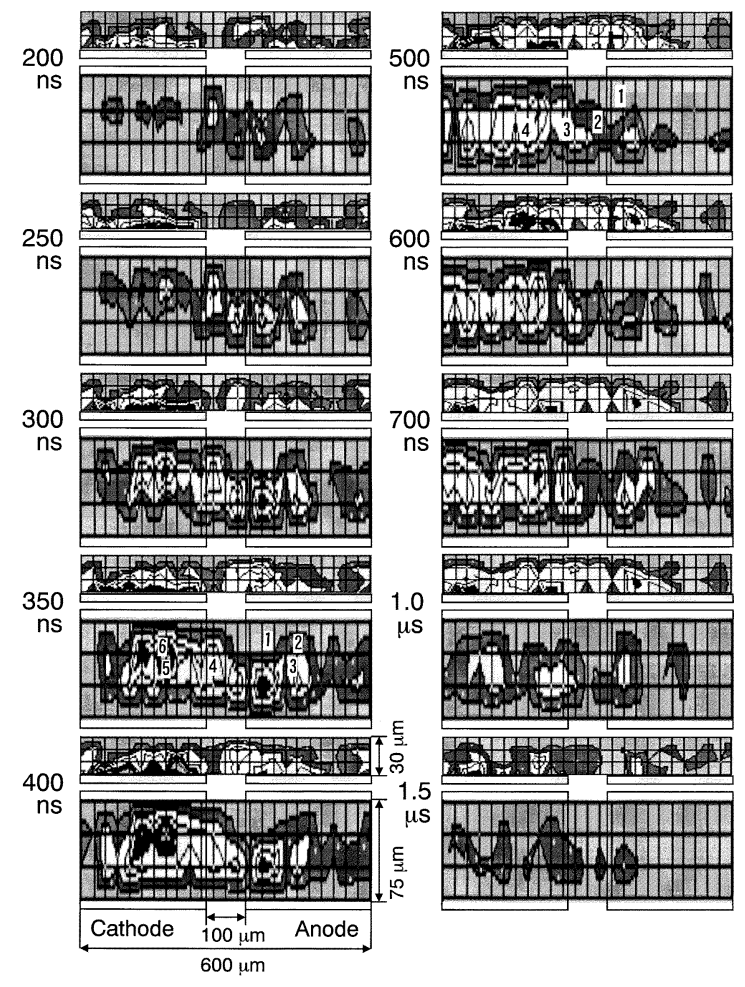

Fig. 7. Spatiotemporal behavior of $\mathrm{Xe}\left(1 \mathrm{~s}_{4}\right)$ atom density at low-voltage $(200 \mathrm{~V})$ mode measured by laser absorption spectroscopy (density scales are $1: 0-0.3 ; 2: 0.3-0.6 ; 3: 0.6-0.9 ; 4: 0.9-1.2 ; 5: 1.2-1.5$; and $6: 1.5-1.8$ in units of $\left.10^{13} \mathrm{~cm}^{-3}\right)$.

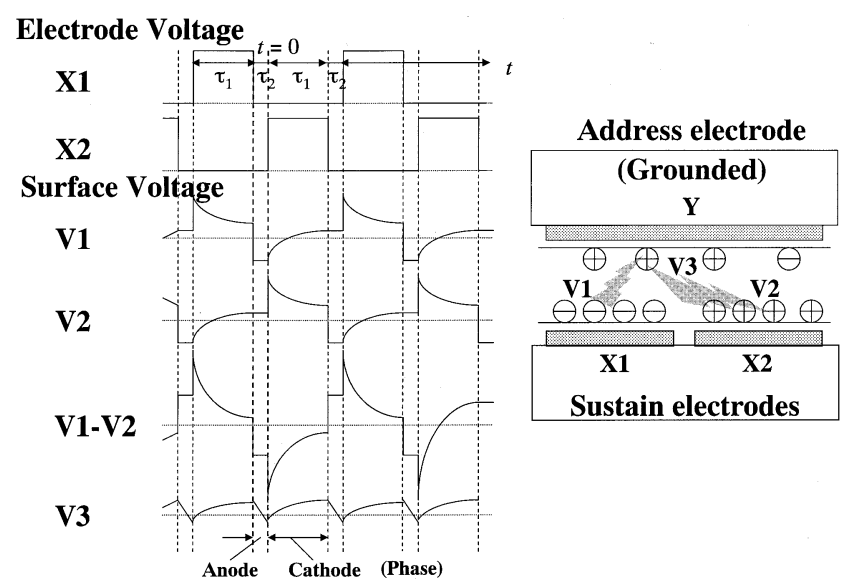

Fig. 8. Schematic illustration of the relation between applied pulses and surface voltages.

than the break-down field, a partial self-erasing discharge may occur between them after the falling-off of the applied voltage when the polarity of $V_{1}$ changes. This causes the decrease of the accumulated charge on $V_{3}$. (The self-erasing discharge may also have a certain delay time, but it is not shown precisely in Fig. 8.) Then, just after the application of next pulse, the main discharge starts with almost no delay between the next temporal anode and the cathode triggered by the self-erasing discharge. At this timing, the surface of address electrode is charged negatively, so that it works as an additional cathode and induces the discharge toward the address electrode. This leads to widening the discharge volume in the high-voltage mode 
operation. However, for the occurrence of this type of coupling between the self-erasing discharge and the main discharge, the interval $\tau_{2}$ between the successive voltage pulses on both the sustain electrodes should be short enough. The necessary condition for this behavior will be examined systematically in near future.

Next, we have to mention that our experiment gives in principle the two sets of 2-D data projected on the $x-y$ and $x-z$ planes, which are integrated over the line-of-sight along $z$ and $y$ directions, respectively, in both the emission and absorption measurements. In that sense, the results cannot be compared directly with the data of 3-D simulations, which are currently being performed extensively [13], [14]. For the purpose, we have to develop a proper algorithm for the 3-D image reconstruction from our experimental data as soon as possible. At the moment, we are preliminarily trying an iterative method [15]. For an example of the crude estimation applied to the data given in Fig. 2, the 3-D peak emission intensity given in Fig. 2 corresponds to the peak values of front and side views multiplied by 1.5 and 2.4, respectively. A similar reconstruction of the absorption data is impossible at the moment due to the smaller number of data points and the larger scattering. If we apply the same multiplication factor to the values of $\mathrm{Xe}\left(1 \mathrm{~s}_{5}\right)$ atom density at the low-voltage mode shown in Fig. 4, the 3-D peak value becomes about $5.3 \times 10^{13} \mathrm{~cm}^{-3}$.

\section{CONCLUSION}

We have succeeded for the first time in performing simultaneous observations from the front and side directions in a realistic ac-type PDP discharge cell. It will make a direct comparison possible between the experimental data and the 3-D simulation results, and help the understandings of the discharge characteristics in newly designed cells with 3-D structures.

\section{ACKNOWLEDGMENT}

The authors would like to thank Y. Wakabayashi of Nandy Electronics for his help in manufacturing the microscopic parts of the test panel, and C. Rauh and S.-L. Blaise, who were short term visiting students at Kyoto University, for programming the computer code for the 3-D image reconstruction.

\section{REFERENCES}

[1] C. Punset, S. Cany, and J.-P. Boeuf, "Addressing and sustaining in alternating current coplanar plasma display panels," J. Appl. Phys., vol. 86, pp. 124-133, 1999.

[2] S. Rauf and M. J. Kushner, "Dynamics of a coplanar-electrode plasma display panel. I. Basic operation; II. Cell optimization.," J. Appl. Phys., vol. 85, pp. 3460-3476, 1999.

[3] Y. Ikeda, J. P. Verboncoeur, P. J. Christenson, and C. K. Birdsall, "Global modeling of a dielectric barrier discharge in $\mathrm{Ne}-\mathrm{Xe}$ mixtures for an alternating current plasma display panel," J. Appl. Phys., vol. 86, pp. 2431-2441, 1999.

[4] J.-H. Seo, W.-J. Yoon, J.-K. Kim, and K.-W. Whang, "Two-dimensional modeling of a surface type alternating current display panel cell: Discharge dynamics and address voltage effects," IEEE Trans. Plasma Sci., vol. 29, pp. 824-831, Mar. 2001.

[5] H.-J. Lee, H.-C. Kim, S.-S. Yang, and J.-K. Lee, "Two-dimensional selfconsistent radiation transport model for plasma display panels," Phys. Plasmas, vol. 9, pp. 2822-2830, 2002.
[6] K. Tachibana, N. Kosugi, and T. Sakai, "Spatio-temporal measurement of excited $\mathrm{Xe}\left(1 \mathrm{~s}_{4}\right)$ atoms in a discharge cell of a plasma display panel by laser spectroscopic microscopy," Appl. Phys. Lett., vol. 65, pp. 935-937, 1994.

[7] K. Tachibana, S. Feng, and T. Sakai, "Spatiotemporal behaviors of excited Xe atoms in unit discharge cell of ac-type plasma display panel studied by laser spectroscopic microscopy," J. Appl. Phys., vol. 88, pp. 4967-4974, 2000.

[8] J.-H. Kim, J.-H. Lee, K.-W. Whang, and Y.-W. Choi, "Temporal behavior of the wall voltage in a surface-type alternating current plasma display panel cell using laser induced fluorescence spectroscopy," $J$. Appl. Phys., vol. 89, pp. 2539-2542, 2001.

[9] Y. Noguchi, A. Matsuoka, K. Uchino, and K. Muraoka, "Direct measurement of electron density and temperature distributions in a micro-discharge plasma for a plasma display panel," J. Appl. Phys., vol. 91, pp. 613-616, 2002.

[10] F. Katafuchi, K. Tachibana, and T. Sakai, "Cross sectional view of spatio-temporal behavior of excited Xe atoms in a discharge cell of ac-type PDP," in Proc. 6th Int. Display Workshops (IDW'99). Sendai, Japan, 1999, pp. 1115-1116.

[11] K. Mizokami, K. Tachibana, N. Kosugi, T. Sakai, and Y. Wakabayashi, "Three dimensional diagnostics of dynamic behaviors of excited atoms in a realistic ac-PDP cell," in Proc. 8th Int. Display Workshops (IDW'01). Nagoya, Japan, 2001, pp. 1755-1756.

[12] G. Oversluizen, S. de Zwart, S. van Heusden, and T. Dekker, "Dependence of PDP efficacy on the gas pressure," in Proc. 7th Int. Display Workshops (IDW'00). Kobe, Japan, 2000, pp. 631-634.

[13] Y. Hirano, Y. Murakami, Y. Takano, and H.-S. Jeong, "3-D computer simulation of Spatio-temporal evolution of discharge from writing to sustaining stage in ac-type PDP cell," in Proc. 8th Int. Display Workshops (IDW'01). Nagoya, Japan, 2001, pp. 889-892.

[14] H.-C. Kim, M.-S. Hur, S.-S. Yang, S.-W. Shin, and J.-K. Lee, "Threedimensional fluid simulation of a plasma display panel cell," J. Appl. Phys., vol. 91, pp. 9513-9520, 2002.

[15] G. Oversluizen, Image Reconstruction from Projections: Fundamentals of Computerized Tomography. New York: Academic, 1980.

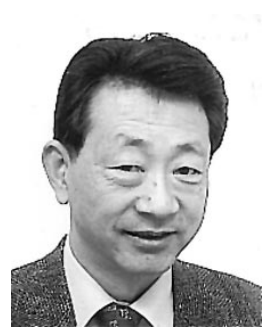

Kunihide Tachibana (M'02) received the B.S. and M.S. degrees in mechanical engineering and the Ph.D. degree from Kyoto University, Kyoto, Japan, in 1968,1970 , and 1973 , respectively.

After one year with Research and Development Center, Toshiba Corporation, Kawasaki, he joined the Department of Electronics, Kyoto Institute of Technology, Kyoto, in 1974. He became a Full Professor in 1988. In the meantime, he worked as a Postdoctoral Research Associate at the Joint Institute for Laboratory Astrophysics, National Institute of Standards and Technology (NIST), and the University of Colorado, Boulder, from 1978 to 1980. In 1995, he became a Full Professor with the Department of Electronic Science and Engineering, Kyoto University. His research interests include plasma diagnostics with lasers and other spectroscopic techniques in various kinds of plasmas for material processing, light sources, and display devices. His current research interests include generation, diagnostics and application of microplasmas of under millimeter scales.

Dr. Tachibana received an award from The Japan Society of Applied Physics (JSAP) in 1995 for the paper "Observation of Coulomb-crystal formation from carbon particles grown in a methane plasma." He has been a Member of the Board of Directors of JSAP since 2001. He is also a Member of American Vacuum Society, The Physical Society of Japan, and several other scientific societies.

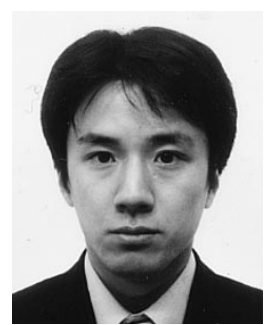

Kaname Mizokami was born in Kyoto, Japan, in 1977. He received the B.S. and M.S. degrees in electronic science and engineering from Kyoto University, Kyoto, in 2000 and 2002, respectively. While he was working toward the M.S. degree, he studied the microdischarge phenomena in a unit cell of plasma display panel (PDP) by laser diagnostics and simulation.

In 2002, he joined Matsushita Electric Industrial Co., Ltd., Takatsuki, Osaka, Japan. He is currently working on PDP technologies. 


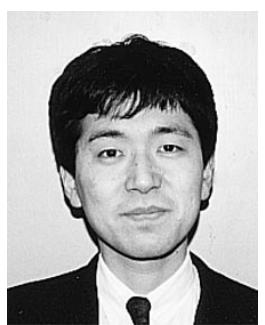

Naoki Kosugi received the B.S. and M.S. degrees in electrical engineering from Kyoto University, Kyoto Japan, in 1987 and 1989, respectively.

$\mathrm{He}$ joined Matsushita Electric Industrial Co., Ltd., Osaka, Japan, in 1989 where he has been working on the research and development of plasma display panels (PDPs). His current interests are on developing ac and dc PDPs with high brightness and efficacy.

Mr. Kosugi is a Member of The Japan Society of Applied Physics.

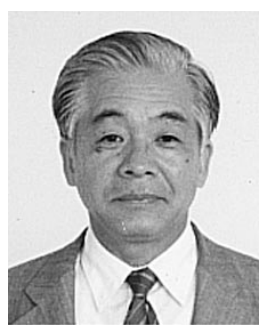

Tetsuo Sakai was born in Tokyo, Japan, in 1939. He received the B.S. degree from Tokyo Denki University, Tokyo, in 1962 and the Ph.D. degree from Tohoku University, Sendai, Japan, in 1994.

He joined the Technical Research Laboratories, NHK (Japan Broadcasting Corporation), Tokyo, in 1957. Until 1967, he was engaged in the development of color TV cameras; he then shifted his research subject to plasma display panels. From 1996 to 1998 , he worked as the Director of PDP Research Laboratories of Hyundai Electronics Japan, Kawasaki, Japan. In 1998, he founded Display Research Laboratories Co., Ltd., and has been working as a Consultant of PDP technology.

Dr. Sakai is a member of the Society for Information Display, the Institute of Image Information and Television Engineers, and the Institute of Electronics, Information, and Communication Engineers. 